\title{
Evaluation of short-circuit current breaking capacity of circuit breaker
}

\author{
SU Yongchun 1, a , WANG xiaoming ${ }^{2, \text { b }}$, ZHOU Ning ${ }^{1, c}$ and SHU Zhan ${ }^{1, d}$ \\ ${ }^{1}$ State grid Jiangxi electric power company Ltd. research institute, China \\ ${ }^{2}$ State grid Jiangxi electric power company Ltd., China \\ asuyongchun@126.com, bwangxm@126.com, czhouning@163.com, dshuzhan@126.com
}

\begin{abstract}
Keywords: Circuit breaker, short-circuit current, dc component.
Abstract. The development of the power grid makes the $\mathrm{X} / \mathrm{R}$ value in the network become more and more large, which leads to the attenuation of the short-circuit current DC component becoming more and more slow when the fault occurs. In this situation, the ability of the breaker to break the short-circuit current is reduced. In this paper, a method of calculating the DC component and its decay time constant of the short-circuit current is given. The DC component of the short-circuit current is obtained by the envelope method, and the least square algorithm is used to calculate the decay time constant. The simulation analysis of the actual breaking capacity of the circuit breaker is given in the example, which provides guidance for the safe and stable operation of the power grid.
\end{abstract}

\section{Introduction}

With the development of our society and the continuous growth of the national economy, the scale of the power grid is getting larger and larger. The voltage level of the power grid is getting higher and higher, the load and power capacity of the power grid are increasing. The increase of the equipment such as the transmission lines and transformers in the power grid makes the structure of the power grid continuously strengthen. On the one hand, it increase the reliability of the power supply, on another hand, it bring the serious problem of short-circuit current exceeding limits [1-4].

Short-circuit current calculation is a basic content of power system simulation and analysis. The short-circuit current level has a great influence on the planning and operation of the power grid (such as the selection of the main wiring, the selection of electrical equipment and conductors, the configuration of circuit breakers, the current limiting measures, etc.), and the protection and control (such as the configuration of various relay protection devices and their parameters setting). In order to ensure the safe and stable operation of the grid, it is necessary to check the breaking capability of the circuit breaker. When the short-circuit current exceed the breaker rated capacity, control measures must be taken such as replacing the circuit breaker, opening the connecting line and adding the current limiting reactor to guarantee the power grid safe operation.

In this paper, the method of evaluating the breaking capacity of the circuit breaker is introduced in detail considering the short-circuit current DC component. After the full current waveform of the short-circuit current is obtained, the wave envelope is used to obtain the DC component. Then the attenuation time constant of the DC component is fitted by the least square method. The calculation method of the influence coefficient of the DC component of the short-circuit current is given. The simulation calculation of the breaking capacity of the circuit breaker is carried out in an example, which provides guidance for the safe and stable operation of the power grid.

\section{Short-circuit current algorithm}

The algorithm of short-circuit current calculation can be divided into two main categories: theoretical algorithms and engineering algorithms. Based on symmetrical component method and compound sequence network, the basic calculation formulas of various short circuit faults are given by the theoretical algorithms. The engineering algorithms are used in engineering applications by specific calculation conditions. There are 4 main engineering algorithms, namely, the equivalent voltage 
source method, the method based on the power flow, the method of relay protection and the calculation curve method.

The equivalent voltage source method is proposed by the IEC60909 standard [5] and is most widely used in the world's large power grid. It is also recommended by IEEE/ANSI related standards. Almost all of the short-circuit current calculation programs such as PSASP, PSD, PSS/E and so on support this method.

The basic principle of the equivalent voltage source method is as the follows:

(1) When short-circuit fault has occurred in power system, a virtual voltage source is introduced at this point, which is the only voltage source.

(2) Other power sources such as synchronous generator, synchronous motor, asynchronous motor and electromotive force of feed network are regarded as 0 . All voltage sources are replaced by its internal impedance.

(3) The Thevenin equivalent algorithm is applied on the short-circuit point, and the system equivalent impedance of the short-circuit point is obtained.

(4) The short-circuit current of the fault point is obtained according to the system equivalent impedance and the virtual voltage source.

In IEC 60909 standards, the equivalent voltage source method are used as Eq. 1 to calculate the three-phase short-circuit current.

$$
I_{k}^{\prime \prime}=\frac{c U_{0}}{\sqrt{3} Z_{k}}
$$

Where $c$ is the voltage coefficient; $U_{0}$ is the nominal voltage of the fault point (rated line voltage); $Z_{k}$ is the positive sequence equivalent impedance of the system on the short-circuit point.

\section{Breaking capacity Evaluation of circuit breaker}

The influences of DC component of short-circuit current. In the current simulation analysis only the fundamental periodic component in the short-circuit current is normally considered. But besides the fundamental periodic component, there also has the DC component. In the past, because of the small size of the power network, the $\mathrm{X} / \mathrm{R}$ value is not very high. In this situation, ignoring the DC component will not cause too much problem to check the breaking capacity of the circuit breaker with the short-circuit current periodic component. However, with the increase of the power system scale, the $\mathrm{X} / \mathrm{R}$ ratio is getting much higher, and the attenuation time constant of the short-circuit current DC component increases gradually. In some cases, only using the periodic component of short-circuit current and neglecting the DC component to check the breaking capacity of the circuit breaker will cause serious problems.

Short-circuit current DC component acquisition. The envelope curve method is used in this paper to obtain the DC component contained in the short-circuit current. As shown in Fig. 1, there are the upper and lower envelopes of the sinusoidal short-circuit current waveform. The average value of the upper and lower envelopes is the DC component curve to be obtained.

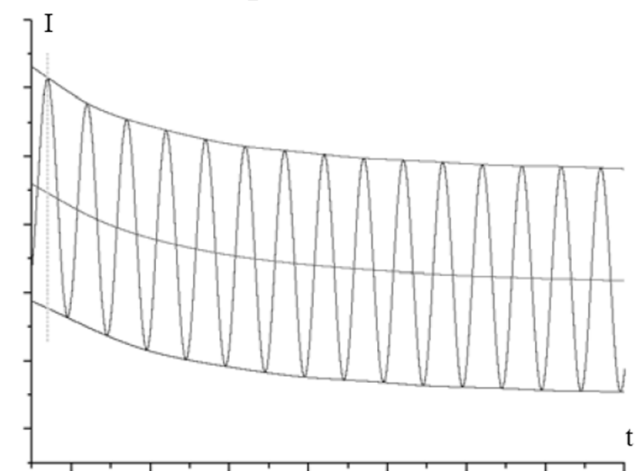

Fig. 1 The envelope method to calculate the DC component. 
Calculation of DC component attenuation time constant. Exponential fitting method is used to calculate the decay time constant of short-circuit current DC component. If the DC component is set as $I(t)$, there is an exponential function $I(t)=A e^{-\frac{t}{T_{d c}}}$. It is necessary to determine the parameter $A$ and time constant $T_{d c}$. In order to calculate conveniently, suppose $A=e^{a},-\frac{1}{T_{d c}}=b(a, b$ is a is the quantity to be desired), then, $I=e^{a+b t}$. The logarithm is taken on both sides of the expression, Thus, $\ln (I)=a+b t$, and let $y=\ln (I)$, it results in $y(t)=a+b t$.

(1) According to the DC component data of short-circuit current, $y_{k}$ is calculated.

$$
y_{k}=\ln \left(I_{k}\right)(k=1,2, \ldots, n)
$$

(2) According to the DC component data, $n$ overdetermined equations are listed with two unknown quantity $a$ and $b$.

$$
a+b t_{k}=y_{k}(k=1,2, \ldots, n)
$$

(3) In Eq. 3, Assuming $X=[a, b]^{T}$, and $Y=\left[y_{1}, y_{2}, \ldots, y_{n}\right]^{T}$, and assuming

$$
A=\left[\begin{array}{cc}
1 & t_{1} \\
1 & t_{1} \\
\cdots & \cdots \\
1 & t_{n}
\end{array}\right]
$$

Eq. 3 can be written as

$$
A X=Y
$$

The least square solution can be obtained from Eq. 5

$$
X=\left(A^{T} A\right)^{-1}\left(A^{T} Y\right)
$$

So parameter $a$ and $b$ are already obtained. Thus the DC component function expression can be written out by the $A$ and time constant $T_{d c}$ determined.

Calculation of circuit breaker breaking capacity. Considering the influence of DC component, the relationship between the actual breaking capacity of the circuit breaker Isc.R and the rated breaking capacity Isc. $N$ is expressed as follows:

$$
\begin{gathered}
I_{s c . R}=I_{s c . N} \sqrt{\frac{1+2 e^{-2 t_{\min } / T_{d c . N}}}{1+2 e^{-2 t_{\min } / T_{d c}}}}=K \cdot I_{s c . N} \\
K=\sqrt{\frac{1+2 e^{-2 t_{\min } / T_{d c . N}}}{1+2 e^{-2 t_{\min } / T_{d c}}}}
\end{gathered}
$$

where

$I_{s c . R}$ - Effective value of the AC component broken by the circuit breaker

$I_{s c . N}$ - The rated breaking current for the circuit breaker

$t_{\min }$ - The shortest break time of the circuit breaker (including time of the main protection plus the opening time)

$T_{d c . N}$ - The standard time constant for the DC component attenuation

$T_{d c}$ - The actual time constant for the DC component attenuation.

Here $K$ is the coefficient considering the influence of the DC component. When the attenuation time constant of the DC component is greater than the standard time constant, there has $K<1$. It mains the actual breaking capacity of the circuit breaker lower than the rated breaking capacity. 
In practical application, the influence coefficient of DC component can be calculated according to Eq. 8, and then the actual breaking capacity of the circuit breaker is calculated according to Eq. 7. When the maximum short-circuit current obtained by simulation calculation is greater than $I_{s c . R}$, it can be judged that the short-circuit current exceed the breaker's capacity. In this case, some measures must be taken to reduce the short-circuit current such as replace the circuit breaker with large capacity one or breaking lines and so on.

\section{Simulation example}

The simulation waveform of short-circuit current of a $220 \mathrm{kV}$ circuit breaker in Jiangxi power grid during bus fault is shown in Fig. 2.

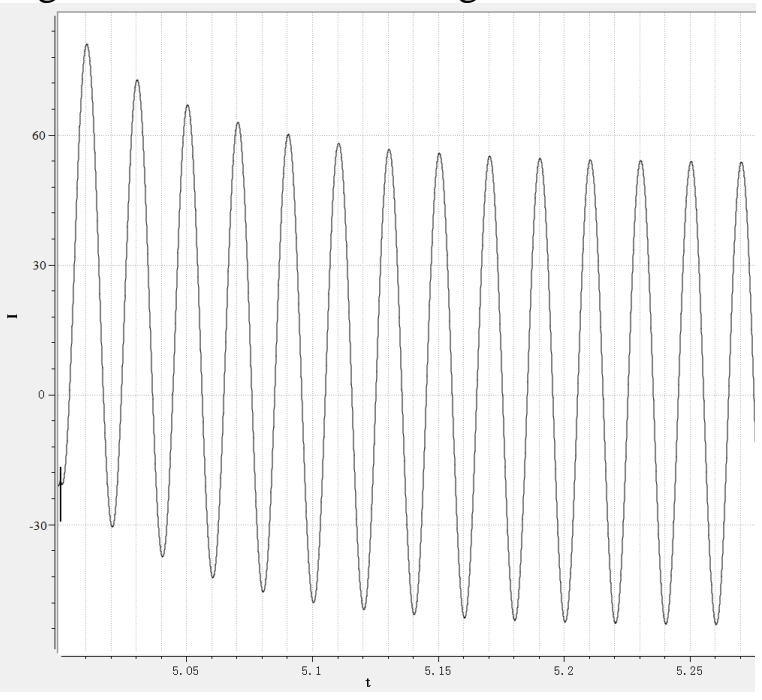

Fig. 2 Short-circuit current waveform

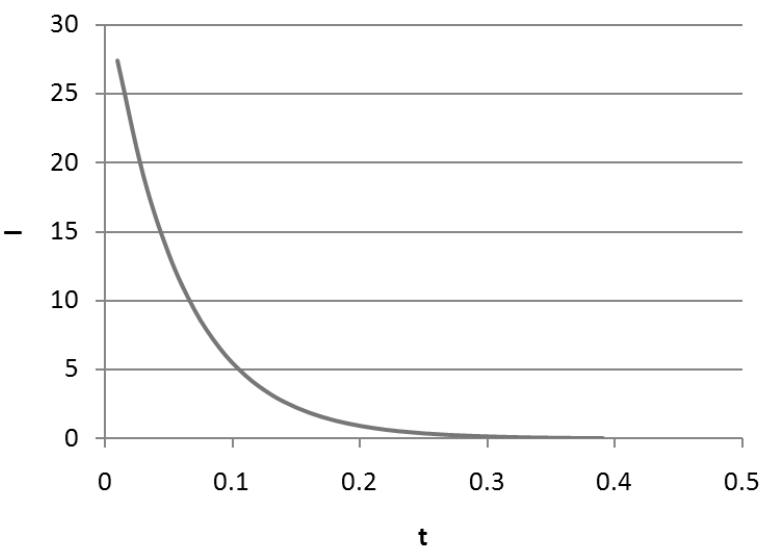

Fig. 3 DC component of the short circuit

As shown in Fig.3, the DC component curve of the short circuit is obtained by the envelope method demonstrated in Fig. 1.

According to Eq. 2-Eq. 6, the DC component decay time constant of short-circuit current can be calculated. The least squares solution of the overdetermined equations can be obtained as $X=(a, b)=(3.49,-17.73)$, and the DC component decay time constant is solved out as $T_{d c}=-\frac{1}{b}=0.0564 \mathrm{~s}=56.4 \mathrm{~ms}$.

According to the regulations of "high voltage AC circuit breaker", the standard DC component decay time constant of $220 \mathrm{kV}$ circuit breaker is $45 \mathrm{~ms}$. According to Eq. 7, assuming the shortest break time of the circuit breaker $t_{\min }$ is $40 \mathrm{~ms}$, it can be calculated that the DC component influence coefficient $K$ is about 0.949 . According to Eq. 7, the actual breaking capacity of the 50kA circuit breaker will decrease to $47.47 \mathrm{kA}$. From Fig. 2, we can see that the fundamental periodic component of the maximum short-circuit current flowing through the circuit breaker is approximately $38.8 \mathrm{kA}$. So although the actual breaking capacity of the circuit breaker has decreased, the maximum short-circuit current is still within the allowable range, and there is no need to take additional short-circuit current limiting measures.

\section{Conclusions}

With the expansion of the power grid scale and the improvement of voltage level, the X/R ratio of the primary equipment increases and the attenuation time constant of the DC component of the short-circuit current increases gradually, which may decrease the actual breaking capacity of the circuit breaker. The problem of short-circuit current DC component in some areas in China is very serious, which leads to the lack of breaking capacity and threaten the safe operation of equipment and 
system. It must be paid more attention. In addition, a large number of restrictive measures such as high impedance transformer and series resistance are adopted in areas with short-circuit current problems. These measures reduce the initial value of the short circuit current, however it increase the decay time constant of the DC component. The simulation calculation show the influence of the short-circuit current DC component. The works in this paper have great significance for the reasonable evaluation of the actual breaking capacity of circuit breakers.

\section{References}

[1] Z.L. LIU and M.L. YIN: Analysis of short-current failures of the $500 \mathrm{kV}$ power system in East China Grid. High Voltage Apparatus, Vol. 44(2008), p.221

[2] J. ZHOU, H. HU, and K.Q. ZHUANG. YIN: Short-circuit current of 500kV East China power grid and its limitation. East China Electric Power, Vol. 34(2006), p.55

[3] L. AI, Y.H. FENG, and W.H. CHEN: Research on short-circuit current problem and limiting measures caused by UHV substation connecting to $500 \mathrm{kV}$ network in Beijing-Tianjin Area and Northern Hebei. Power System Protection and Control, Vol. 45(2017), p.133

[4] S.L. YAO, and H. TIAN: A Comparison between BPA and PSASP Software Programs for Calculating Short-circuit Currents. Automation of Electric Power Systems, Vol. 35(2011), p.112

[5] IEC 60909: Short-circuit currents in three-phase AC systems. International standard (2001). 